\title{
Commentary
}

\section{Ontogeny of ADME Processes during Postnatal Development in Man and Preclinical Species: A Comprehensive Review}

\author{
Luc M. De Schaepdrijver, Pieter P. J. Annaert, and Connie L. Chen \\ Janssen R\&D, Beerse, Belgium (L.M.D.S.); University of Leuven, Leuven, Belgium (P.P.J.A.); and Health and Environmental \\ Science Institute, Washington, DC (C.L.C.)
}

Received September 7, 2018; accepted December 14, 2018

Substantial differences can exist in the absorption, distribution, metabolism, and excretion (ADME) of orally and/or systemically administered medicines depending on the age of patients or animals, leading to potential differences in drug efficacy and toxicity. These differences are usually most prominent in the neonatal period and early infancy. Likewise, maturational changes in the physiology of the gastrointestinal tract (GIT), liver, and renal systems can impact the ADME parameters and result in rapidly changing systemic exposures with progressing age.

Historically, very few new therapies are developed specifically for neonates, in whom most drugs are used "off-label" (Conroy and McIntyre, 2005; Cuzzolin et al., 2006). Several efforts are underway to increase our knowledge in the neonatal spectrum (https://c-path.org/ programs/inc/; Davis-Bruno et al., 2016; Ward et al., 2017). Following the implementation of pediatric regulations by the US Food and Drug Administration (Pediatric Research Equity Act, 2003 made permanent under FDA Safety and Innovation Act, 2012) and the European Commission (Pediatric Regulation No 1901/2006), an agreed-upon Pediatric Study Plan and Pediatric Investigational Plan are needed before the submission of a New Drug Application or Marketing Authorization Application in the United States and European Union. These regulations have been essential in stimulating the conduct of well-controlled pediatric clinical trials earlier in development (ICH E11(R1), 2017).

For the design of juvenile animal studies, often performed in support of pediatric drug development, it is critical to select the appropriate species and age relative to the age of the intended pediatric population and the target organs of concern (ICH S11, 2018). A strong understanding of comparative organ development between humans and laboratory animals is therefore essential. This encompasses not only structural and functional components of development but also the ontogeny of ADME parameters. In addition, insights into the mechanisms underlying (agedependent) drug disposition in neonates and juvenile animals is required to ensure adequate understanding of the results obtained.

The Health and Environmental Sciences Institute recently launched a multisector collaborative research effort to increase the knowledge base in the nonclinical neonatal space to better inform decisions made in the clinic. This program brings together an international multidisciplinary team from academia, industry, and government to address the gaps in neonatal drug development through distinct, but interconnected, projects (Davis-Bruno et al., 2016). As an important first step, literature was reviewed by expert groups on the ontogeny of ADME-related processes in man and preclinical species for the GIT, liver, kidney, brain, and heart. The review paper on GIT is included in the current issue of Drug Metabolism and Disposition (Neal-Kluever et al., 2019), whereas additional reviews are forthcoming.

When using pharmacokinetic modeling as a physiological basis, the availability of high-resolution ontogeny profiles of drug-metabolizing enzymes and transporters will support better prediction of drug disposition in neonates and infants. In addition, better insight into the ontogeny profiles of the corresponding proteins in preclinical species may prove instrumental for selecting the appropriate model and starting age for juvenile animal toxicity studies. Unfortunately, knowledge of the cross-species ontogeny of GIT, hepatic, and renal drug-metabolizing enzymes, transporters, and nuclear receptors in neonatal animals and humans currently remains incomplete. The purpose of this series of comprehensive reviews was to compile the available ex vivo ontogeny data in this domain and pinpoint knowledge gaps and inconsistencies. Directions for further research as they relate to each organ system are provided.

\section{References}

Conroy S and McIntyre J (2005) The use of unlicensed and off-label medicines in the neonate Semin Fetal Neonatal Med 10:115-122.

Cuzzolin L, Atzei A, and Fanos V (2006) Off-label and unlicensed prescribing for newborns and children in different settings: a review of the literature and a consideration about drug safety. Expert Opin Drug Saf 5:703-718.

Davis-Bruno K, De Schaepdrijver L, Annaert P, Campion S, Carleer J, Chapin R, Harrouk W, Laffan S, McCune S, Moffat Graeme J, et al. (2016) Nonclinical models for neonatal pediatric drug development. Reprod Toxicol 64:49.

ICH E11(R1) (2017) ICH E11(R1) guideline on clinical investigation of medicinal products in the paediatric population, Step 5. European Medicines Agency, London, United Kingdom.

ICH S11 (2018) ICH guideline S11 on nonclinical safety testing in support of development of paediatric medicines, Step 2b. European Medicines Agency, London, United Kingdom.

Neal-Kluever A, Fisher J, Grylack L, Kakiuchi-Kiyota S, and Halpern W (2019) Physiology of the Neonatal Gastrointestinal System Relevant to the Disposition of Orally Administered Medications. Drug Metab Dispos 47:296-313.

Ward RM, Benjamin D, Barrett JS, Allegaert K, Portman R, Davis JM, and Turner MA; International Neonatal Consortium (INC) (2017) Safety, dosing, and pharmaceutical quality for studies that evaluate medicinal products (including biological products) in neonates. Pediatr Res 81:692-711

Address correspondence to: Dr. Luc De Schaepdrijver, Non Clinical Safety, Janssen R\&D, Turnhoutseweg 30, B-2340 Beerse, Belgium. E-mail: Idschaep@its. jnj.com 